\title{
Prevalencia de divertículos colónicos asintomáticos en población chilena como hallazgo imagenológico en tomografía de abdomen y pelvis
}

\author{
Natalia Moreno B. ${ }^{1}$, Viviana Saavedra J. ${ }^{2}$, Tamara Peters A. ${ }^{2}$, Nicolas von Jentschyk R. ${ }^{2}$, \\ Günther Bocic A. ${ }^{3}$, Antonella Sanguinetti M. ${ }^{3}$, Mario Abedrapo M. ${ }^{3}$, José Luis Llanos B. ${ }^{3}$, \\ Rodrigo Azolas C. ${ }^{3}$, Mauricio Díaz B. ${ }^{3}$, Hernán Soto SM. ${ }^{2}$ y Felipe Bocic W. ${ }^{3}$
}

'Departamento de Cirugía

Hospital Clínico Universidad

de Chile. Santiago, Chile.

${ }^{2}$ Servicio de Radiología

Hospital Clínico Universidad

de Chile. Santiago, Chile.

${ }^{3}$ Interno Facultad de Medicina

Universidad de Chile. Santiago, Chile.

Recibido el 22 de mayo de 2018 y aceptado para publicación el 7 de agosto de 2018

Correspondencia a: Dra. Natalia Moreno B. anatalia96@gmail.com

Prevalence of asymptomatic colonic diverticula in Chilean population as an imaging finding in abdominal and pelvic tomography

Introduction: Asymptomatic colonic diverticular prevalence varies in the general population between 20 and $60 \%$ in international references, however, we couldn`t find statistics about it in national reports. A method for detection of this nosological entity is the abdomen and pelvis computed tomography.

Aim: Determine the prevalence of asymptomatic colonic diverticulosis in the general population as a find in abdomen and pelvis computed tomography (TCAP). Materials and Method: Cross-sectional study with descriptive statistics, selecting patients with no history of diverticulosis, who underwent TCAP in our Hospital. 1,449 cases are reviewed and 1,177 are included. Results: The general prevalence of colonic diverticula was $28.3 \%$. Their presence increases with age, being close to $60 \%$ in $>80$ years. Almost $90 \%$ are located in the left colon, being more frequent the right side diverticulosis in population under 60 years. ConclusionsB The overall prevalence of asymptomatic colonic diverticulosis, detected as a finding in TCAP, corresponds to about one third of patients.

Key words: colonic diverticulum; asymptomatic; prevalence.

\section{Resumen}

Introducción: La prevalencia de diverticulosis colónica en población general varía entre 20-60\% según referencias internacionales, sin embargo, hay escasos datos nacionales. Un buen método para la detección de esta entidad es la tomografía computarizada de abdomen y pelvis (TACP). Objetivos: Determinar la prevalencia de diverticulosis colónica en población general como hallazgo en tomografías computarizadas de abdomen y pelvis. Materiales y Método: Estudio de corte transversal con estadística observacional, seleccionando pacientes sin antecedentes de diverticulosis que se realizaron TCAP en un hospital clínico en un periodo de 3 meses. Se revisan 1.449 TCAP y se incluyen 1.177 casos. Resultados: La prevalencia general de divertículos colónicos fue de $28,3 \%$, esta cifra aumenta con la edad alcanzando un $60 \%$ en mayores de 80 años. Casi un $90 \%$ se localizan en colon izquierdo, y los casos en lado derecho se acumulan en menores de 60 años. Conclusiones: La prevalencia global de diverticulosis, detectadas por TCAP en esta población, corresponde a cerca del $30 \%$ de los pacientes.

Palabras clave: divertículo de colon; asintomático; prevalencia.

\section{Introducción}

La diverticulosis de colon corresponde a la alteración anatómica colónica más frecuente. Se define como la herniación de mucosa y submucosa colónica a través de una zona de debilidad de la capa muscu- lar, coincidente con sitios de penetración de los vasos rectos en la capa muscular circular, en el caso de los llamados pseudodivertículos, o una herniación de todas las capas intestinales en los verdaderos ${ }^{4,5}$. La diverticulosis como tal corresponde a la portación asintomática; cuando se presentan síntomas habla- 
mos de enfermedad diverticular. Su importancia radica en que esta herniación puede sufrir complicaciones tales como perforación, fístula, obstrucción o sangrado con presentación clínica variable, desde casos leves de manejo domiciliario hasta casos muy graves que requieren una cirugía de urgencia, con una mortalidad estimada de 2,5 por 100.000 pacientes al año ${ }^{6}$. Aproximadamente un $20 \%$ de los portadores de divertículos sufrirán complicaciones con cuadros clínicos específicos, la mayoría de ellos leves y de buena respuesta a tratamiento médico ${ }^{7-9}$, sin embargo, algunos estudios con diagnóstico por colonoscopía y seguimiento prospectivo informan bajas tasas de complicación, incluso $1 \%$ a 10 $a_{n}{ }^{10,11}$. Aun cuando no está claramente definido cuál sería el mecanismo fisiopatológico que participa en la génesis de los divertículos, existen teorías que lo relacionan con la hiperactividad contráctil del colon, la que provocaría un incremento de la presión en segmentos cerrados, causando una herniación de la mucosa. Dicho fenómeno de tipo mecánico se vería favorecido por factores alimenticios, étnicos y por alteraciones del tejido conectivo y muscular ${ }^{11,13}$.

Respecto a los factores de riesgo para presentar diverticulosis, en todos los reportes la edad es el factor más importante, pero se pueden mencionar otros como obesidad, alteraciones congénitas del colágeno, tabaquismo, dieta baja en fibra, dismotilidad intestinal y herencia genética ${ }^{8}$.

Los divertículos pueden encontrarse en cualquier segmento colónico, en occidente son más frecuentes en el colon sigmoides $(95 \%)$ y corresponden a pseudodivertículos, mientras que en los países asiáticos resultan ser más frecuentes en el colon derecho con características anatómicas de divertículos verdade$\operatorname{ros}^{14-16}$

Los reportes internacionales señalan fuertemente que su prevalencia incrementa con la edad, encontrando desde un $20 \%$ en la cuarta década de la vida, hasta un $60 \%$ en la sexta ${ }^{17,18}$, esto es concordante con reportes nacionales, aunque se trata de series pequeñas ${ }^{19}$ o con métodos diagnósticos previos al uso de TC.

Respecto a la distribución por género, esta resulta ser bastante homogénea, con una leve predominancia masculina en los grupos más jóvenes, situación que se invierte al avanzar la edad ${ }^{3,7}$.

La mayoría de los estudios de prevalencia de diverticulosis están basados en colonoscopía, necropsias y enema baritado. Antes de la era del TC, el enema baritado fue el estudio más utilizado en pacientes sintomáticos ${ }^{20}$. Si bien es cierto que el estudio endoscópico es un muy buen método de diagnóstico y, además, en ocasiones terapéutico, tie- ne la desventaja de requerir una preparación previa no siempre bien tolerada y, además, presentar morbilidad asociada por ser un método invasivo (tasa de perforación 1 por cada 1.000$)^{21}$. Por otro lado, la tomografía computada de abdomen y pelvis (TCAP) no es invasiva y su tasa de morbilidad es menor, convirtiéndose en los últimos años en el examen de elección para tamizaje de cáncer colorrectal y otras patologías colónicas por su excelente precisión. La utilidad del TCAP está ampliamente documentada en la detección de divertículos; si bien su mayor uso es en los casos de diverticulitis aguda ${ }^{2,22}$, y más aún, en casos complicados con abscesos ${ }^{22}$, la utilidad del TCAP para detectar diverticulosis no complicada es alta, alcanzando una sensibilidad y especificidad mayores al $90 \%{ }^{23}$.

Existe escasa evidencia nacional que reporte la prevalencia de diverticulosis colónica asintomática por $\mathrm{TC}^{24,25}$, por lo que se decide presentar esta experiencia.

\section{Materiales y Método}

Para desarrollar esta investigación se utilizó metodología cuantitativa observacional, con corte transversal y estadística analítica.

Se incluyeron pacientes mayores de 15 años, consecutivos, que se realizan un TCAP de abdomen y pelvis de urgencia o electiva, por cualquier causa que no incluyera sospecha de patología de colon. Se excluyeron aquellos pacientes con estudio dirigido por antecedentes o sospecha de enfermedad diverticular, cirugía colónica previa por esta patología, informe incompleto y estudios repetidos de un mismo paciente.

Se analizaron TCAP realizadas en el Servicio de Radiología del Hospital Clínico de la Universidad de Chile entre el 1 de enero y el 31 de marzo del año 2016. Se utilizó un equipo Siemens Somation $64^{\circledR}$. La técnica fue similar para todos los pacientes, que estaban en ayunas de al menos 4 horas, recibieron una inyección endovenosa de $2 \mathrm{ml} / \mathrm{kg}$ de medio de contraste (Optiray ${ }^{\circledR} 320$ ) a un promedio de $4 \mathrm{ml} /$ seg en $50 \mathrm{ml}$. de solución fisiológica. El estudio se realizó con colimación 24 X 1,2 mm, cortes de 5 $\mathrm{mm}$ con incremento de $1,2 \mathrm{~mm}$ y voltaje de $120 \mathrm{KV}$, reconstrucción coronal y sagital con un grosor de $2,5 \mathrm{~mm}$. Los resultados fueron informados por 2 radiólogos de abdomen, staff de esta unidad, con más de 5 años de experiencia. Se informa la presencia/ ausencia de divertículos y la distribución anatómica por segmentos (ascendente, transverso, descendente, sigmoides). Si los divertículos estaban en más de un segmento se especificó: "sigmoides + descendente", 
Tabla 1. Distribución por sexo de todos los pacientes incluidos al estudio

\begin{tabular}{|lccc|}
\hline & $\begin{array}{c}\text { Total } \\
(\mathbf{n}=\mathbf{1 . 1 7 7})\end{array}$ & $\begin{array}{c}\text { Masculino } \\
(\mathbf{n}=\mathbf{5 4 1})\end{array}$ & $\begin{array}{c}\text { Femenino } \\
(\mathbf{n}=\mathbf{6 3 6})\end{array}$ \\
\hline TCAP $(+)$ & 334 & $154(46,1 \%)$ & $180(53,9 \%)$ \\
TACP $(-)$ & 843 & $387(46 \%)$ & $456(54 \%)$ \\
\hline
\end{tabular}

TCAP: Tomografía computarizada de abdomen y pelvis. $(+)$ : Positivo para diverticulosis. (-): Negativo para diverticulosis.

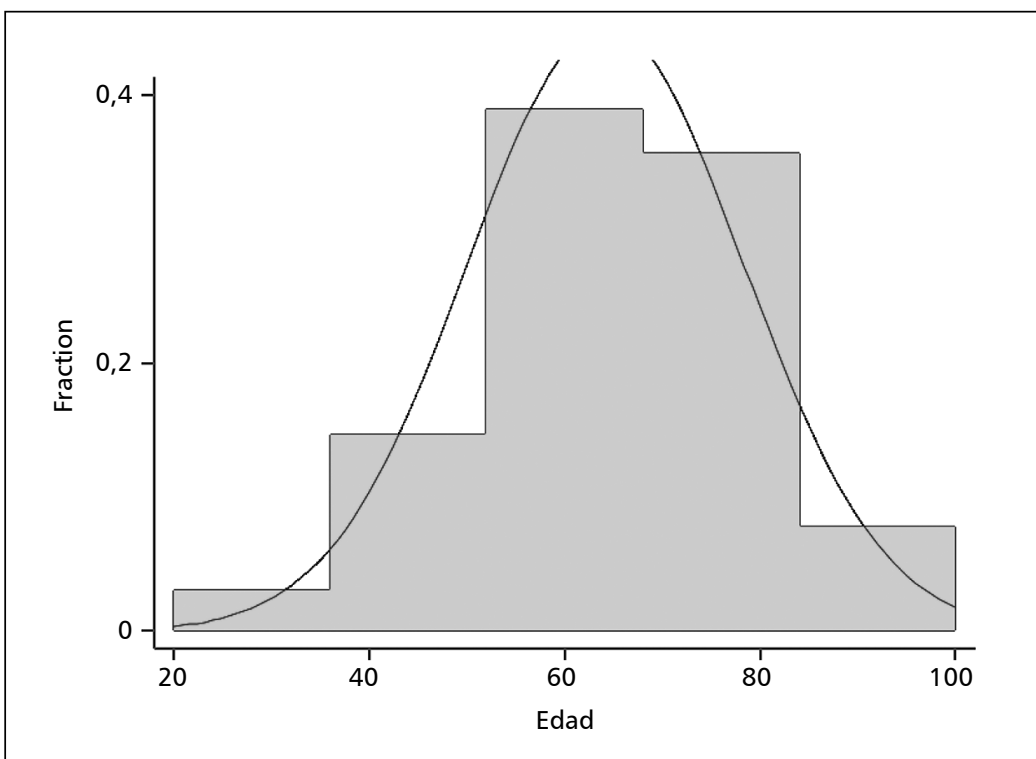

Figura 1. Gráfico de barras que muestra la distribución de la edad de los pacientes con divertículos. Esta muestra tiene una distribución no normal.

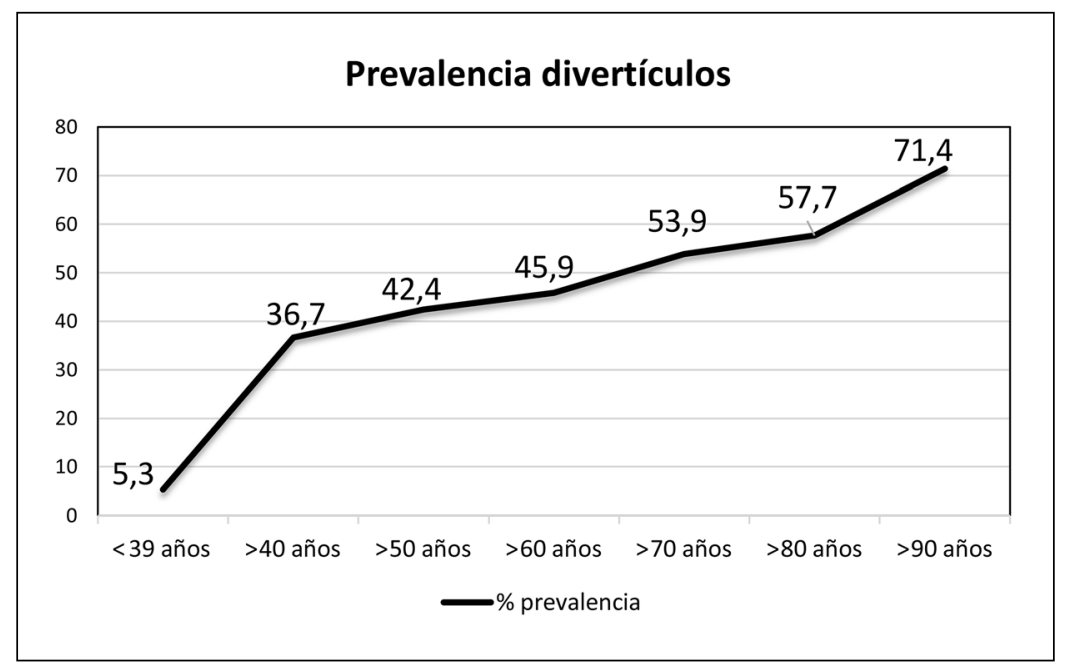

Figura 2. Gráfico que muestra la prevalencia creciente de divertículos según rango etario. "sigmoides + descendente + transverso" o "pancolónico". También se describen por lateralidad (Derecho: ciego y colon ascendente. Izquierdo: sigmoides hasta colon transverso).

Se decide no utilizar escalas de severidad basados en cantidad de divertículos.

De 1.449 TCAP analizados, 1.177 cumplieron los criterios de inclusión y exclusión. Se tabularon datos demográficos de los pacientes seleccionados respecto a la edad y se efectuó una segmentación por decenios, además se dividió al grupo en mayores o menores de 60 años (tercera edad). Para el análisis de datos y cálculos estadísticos se utilizó el programa Stata 10. Se utilizó estadística descriptiva estableciendo significancia con un $\mathrm{p}<0,05$.

\section{Resultados}

En el análisis de la muestra se observa una prevalencia general de diverticulosis de $28,4 \%$ (de 1.177 TCAP examinados, 334 fueron positivos para divertículos), de estos un 53,9\% correspondió a sexo femenino (Tabla 1). No hubo diferencias significativas respecto al sexo entre los pacientes con TCAP positivos $v s$ negativos para divertículos $(\mathrm{p}=0,78)$. El promedio de edad de la muestra fue de 56,2 años (rango 15-97 años). La edad promedio del grupo con divertículos fue significativamente mayor que el grupo sin el hallazgo (64,2 vs 48,3 años $\mathrm{p}<0,0001)$. Se comprueba que la distribución de la edad de los grupos no es normal mediante el test de ShapiroWilk ( $\mathrm{z}=0,0081)$ (Figura 1).

No hubo hallazgo de divertículos bajo los 20 años y se aprecia un incremento progresivo de la prevalencia al avanzar la edad, acumulándose sobre los 60 años el $61 \%$ de los casos con divertículos (Tabla 2, Figura 2).

En nuestra serie estudiada un 92,2\% tuvo divertículos presentes sólo en el colon izquierdo, un 1,8\%

Tabla 2. Prevalencia acumulada de divertículos por rango etario en decenios

\begin{tabular}{|cc|}
\hline Edad & Prevalencia \\
$<39$ años & $5,3 \%$ \\
$\geq 40$ años & $36,7 \%$ \\
$\geq 50$ años & $42,4 \%$ \\
$\geq 60$ años & $45,9 \%$ \\
$\geq 70$ años & $53,9 \%$ \\
$\geq 80$ años & $57,7 \%$ \\
$\geq 90$ años & $71,4 \%$ \\
\hline
\end{tabular}

Se ve un aumento en la prevalencia a mayor rango etario. 
tuvo divertículos sólo en el colon derecho y en un $5,9 \%$ de los casos esta patología tuvo localización pancolónica. Si se suman los casos pancolónicos y los exclusivos de colon derecho, podemos decir que se encontraron divertículos al lado derecho en un 7,8\% de los pacientes. La localización por segmentos y patrones se resumen en las Tablas 3 y 4 , Figuras 3. La edad no parece influir en la localización ya que se descarta una correlación entre edad y localización por test de Spearman $(\mathrm{t}=0,08)$ (Tabla 5 , Figura 4). Se observa un promedio de edad menor en el grupo de pacientes que presentó divertículos en el colon ascendente, aunque esto no es significativo. Tampoco se observa una correlación entre el sexo y la localización de los divertículos (Tablas 6 y 7), pero sí entre el sexo y la edad de presentación, ya que se encontró una predominancia femenina en los pacientes con divertículos sobre los 70 años de edad $((\mathrm{p}=0,002)$ y predominio masculino en los menores de 40 años $(\mathrm{p}=0,03)$ (Tabla 8). Cabe destacar que el grupo con divertículos tuvo predominancia femenina al avanzar la edad (Mann-Whitney $=0,0046)$ lo que corrobora el hallazgo anterior (Tabla 9).

\section{Discusión}

La diverticulosis colónica es una alteración anatómica que resulta ser, en la gran mayoría de los casos, asintomática o asociada a sintomatología inespecífica, muchas veces mal catalogada como trastorno digestivo funcional ${ }^{9,26}$. Es relevante cono- cer las características epidemiológicas de nuestra población, con el fin de mejorar el proceso diagnóstico y terapéutico en sintomáticos y estimar la prevalencia esperada en asintomáticos, en un país aun sin tamizaje establecido. Este trabajo representa una de las primeras investigaciones locales de prevalencia

Tabla 3. Prevalencia de divertículos según segmento colónico afectado

\begin{tabular}{|cc|}
\hline Segmento colónico afectado & Prevalencia \\
\hline Sigmoides & $309(92,5 \%)$ \\
\hline Descendente & $221(66,1 \%)$ \\
\hline Transverso & $79(23,6 \%)$ \\
\hline Ascendente & $26(7,8 \%)$ \\
\hline
\end{tabular}

Tabla 4. Prevalencia de divertículos según patrones de distribución anatómica

\begin{tabular}{|c|c|c|}
\hline Patrones de distribución anatómica & Prevalencia & \\
\hline Sólo sigmoides & $107 \quad(32 \%)$ & \multirow{4}{*}{$\begin{array}{l}\text { Colon izquierdo } \\
308(92,2 \%)\end{array}$} \\
\hline Sólo colon descendente & $19(5,7 \%)$ & \\
\hline Sigmoides + descendente & $125(37,4 \%)$ & \\
\hline Sigmoides + descendente + transverso & $57 \quad(17 \%)$ & \\
\hline Pancolónico & $20 \quad(6 \%)$ & \multirow{2}{*}{$\begin{array}{l}\text { Colon derecho } \\
26(7,8 \%)\end{array}$} \\
\hline \multirow[t]{2}{*}{ Sólo colon ascendente } & $6(1,8 \%)$ & \\
\hline & $334(100 \%)$ & \\
\hline
\end{tabular}

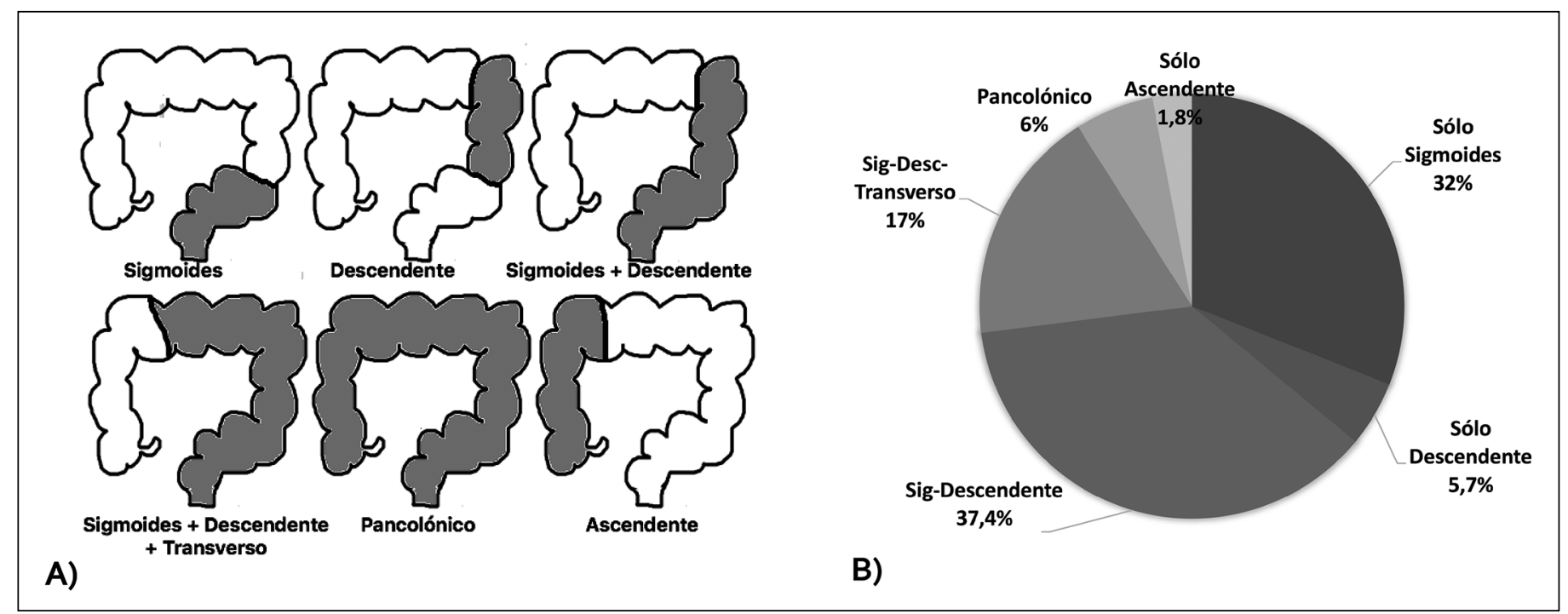

Figura 3. A) Patrones de localización anatómica. B) Gráfico que esquematiza la prevalencia de divertículos según patrones de localización anatómica. 


\section{ARTÍ́CULO ORIGINAL}

Tabla 5. Distribución de pacientes con divertículos según edad y localización anatómica

\begin{tabular}{|llccc|}
\hline Edad & $\begin{array}{c}\text { Colon izquierdo } \\
(\mathbf{n}=\mathbf{3 0 8})\end{array}$ & $\begin{array}{c}\text { Colon derecho } \\
(\mathbf{n}=\mathbf{6})\end{array}$ & $\begin{array}{c}\text { Pancolónico } \\
(\mathbf{n}=\mathbf{2 0})\end{array}$ \\
$<60$ años & $<39$ años & 14 & 1 & 1 \\
$(\mathrm{n}=130)$ & $>40$ años & 34 & 1 & 2 \\
& $>50$ años & 69 & 2 & 6 \\
$>60$ años & $>60$ años & 71 & 1 & 3 \\
$(\mathrm{n}=204)$ & $>70$ años & 77 & 1 & 4 \\
& $>80$ años & 41 & 0 & 1 \\
\hline
\end{tabular}

Figura 4. Gráfico de cajas que ilustra la distribución de edad según distintos patrones de distribución anatómica. Cada caja representa los percentiles 10,50 y 90 , la DS y los casos extremos. Los números del eje $\mathrm{x}$ representan: 1: solo sigmoides; 2: solo descendente; 3 : sigmoides + descendente; 4: sigmoides + descendente + transverso; 5 : pancolónico; 6: solo ascendente.

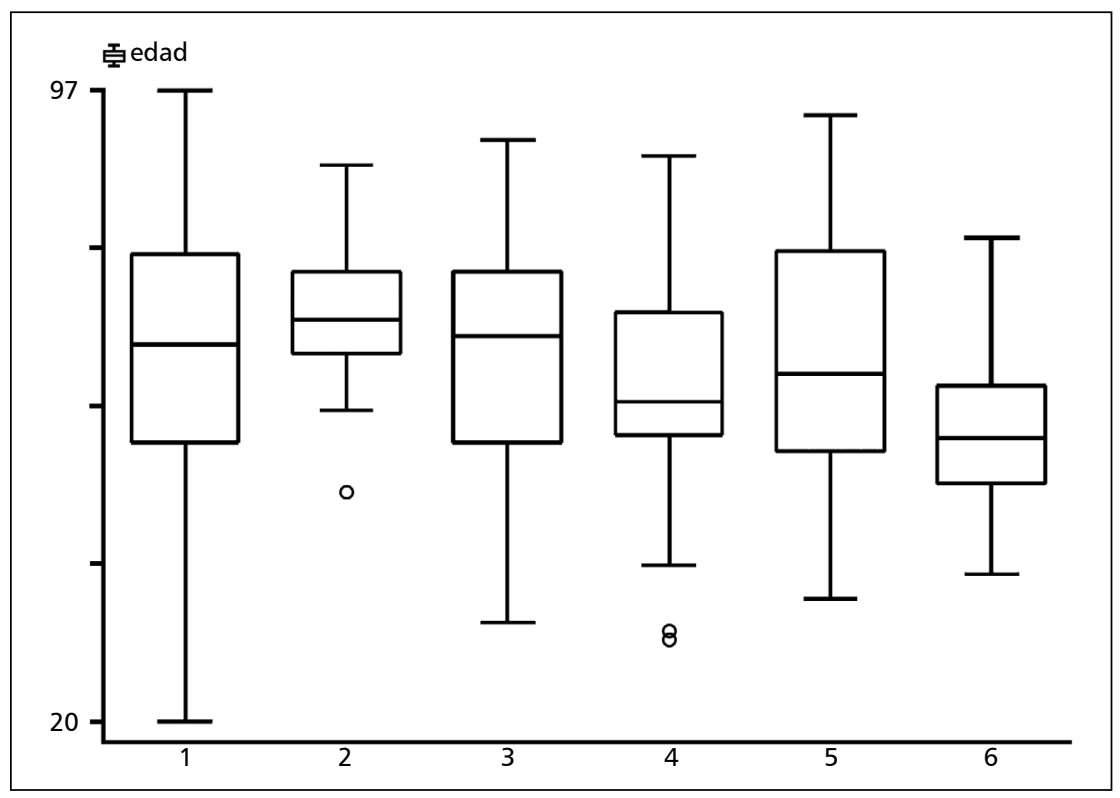

Tabla 6. Distribución de pacientes con divertículos según segmento colónico afectado y sexo

\begin{tabular}{|lcccc|}
\hline Segmento afectado & $\mathbf{N}^{\mathbf{0}}$ veces afectado & Masculino & Femenino & $\boldsymbol{\chi}^{\mathbf{2}}$ \\
\hline Sigmoides & 309 & 141 & 168 & 0,37 \\
\hline Descendente & 221 & 106 & 115 & 0,9 \\
Transverso & 79 & 35 & 44 & 0,13 \\
Ascendente & 26 & 13 & 13 & 0,04 \\
\hline
\end{tabular}

Tabla 7. Distribución de pacientes con divertículos según distintos patrones de localización anatómica y sexo

\begin{tabular}{|lcccc|}
\hline Patrones de localización anatómica & $\begin{array}{c}\text { Total } \\
(\mathbf{n = 3 3 4 )}\end{array}$ & $\begin{array}{c}\text { Masculino } \\
(\mathbf{n = 1 5 4 )}\end{array}$ & $\begin{array}{c}\text { Femenino } \\
(\mathbf{n = 1 8 0 )}\end{array}$ & $\boldsymbol{\chi}^{\mathbf{2}}$ \\
\hline Sólo sigmoides & 107 & 46 & 61 & 0,62 \\
Sólo colon descendente & 19 & 11 & 8 & 1,13 \\
Sigmoides + descendente & 125 & 61 & 64 & 0,75 \\
\hline Sigmoides + descendente + transverso & 57 & 23 & 34 & 0,42 \\
Pancolónico & 20 & 11 & 9 & 0,35 \\
Sólo colon ascendente & 6 & 2 & 4 & $0,05^{*}$ \\
\hline
\end{tabular}

*Se utilizó corrección de Yates al $\chi^{2}$ por la baja frecuencia de los casos. 
ARTíCULO ORIGINAL

Tabla 8. Distribución de los 1.177 pacientes según edad y sexo

\begin{tabular}{|lcccc|}
\hline Grupo etario & $\begin{array}{c}\text { Total } \\
(\mathbf{n}=\mathbf{1 . 1 7 7})\end{array}$ & $\begin{array}{c}\text { Masculino } \\
(\mathbf{n}=\mathbf{5 4 1})\end{array}$ & $\begin{array}{c}\text { Femenino } \\
(\mathbf{n}=\mathbf{6 3 6})\end{array}$ & Valor p \\
\hline $1-19$ años & $0 \%$ & $0 \%$ & $0 \%$ & 0,96 \\
\hline $20-29$ años & $1,8 \%$ & $1,72 \%$ & $1,85 \%$ & $0,03 *$ \\
\hline $30-39$ años & $8,8 \%$ & $14,29 \%$ & $4,49 \%$ & 0,25 \\
\hline $40-49$ años & $18,3 \%$ & $21,74 \%$ & $15,52 \%$ & 0,49 \\
\hline $50-59$ años & $35,4 \%$ & $37,74 \%$ & $33,33 \%$ & 0,47 \\
$60-69$ años & $36,6 \%$ & $39,18 \%$ & $34,26 \%$ & $0,02 *$ \\
\hline $70-79$ años & $52 \%$ & $41,89 \%$ & $61,25 \%$ & 0,5 \\
\hline 80 años & $56,4 \%$ & $53,12 \%$ & $60,38 \%$ & \\
\hline
\end{tabular}

La edad se muestra por decenios. Se comparan los porcentajes de mujeres y hombres por cada decenio considerando significativo el p $<0,05$.

de diverticulosis en asintomáticos, con diagnóstico por TC, con más de 1.000 pacientes.

La prevalencia global observada en nuestro estudio $(28 \%)$ es similar a la reportada en algunas series europeas de hace 10 años atrás ${ }^{27-29}$, sin embargo, es menor a la informada por estudios europeos más recientes $^{30}$, que reflejan un aumento de esta patología en Europa por el envejecimiento de la población. Además, nuestros resultados muestran un aumento de la prevalencia con la edad, lo que es concordante con la literatura que describe ampliamente este fenómeno. Este hecho se ha atribuido a un deterioro estructural de la pared muscular en la población de mayor edad ${ }^{1,7,9,31}$. La prevalencia en pacientes jóvenes menores de 40 años fue de $5,3 \%$, lo que es discordante con reportes recientes que muestran hasta un $25 \%$ de pacientes jóvenes con divertículos colónicos $^{6}$, asemejándose nuevamente a estudios previos que reportan $5-30 \%$ de prevalencia ${ }^{32-34}$.

Respecto a la ubicación anatómica, de manera similar a la literatura internacional anglosajona y europea, colon sigmoides y/o descendente acumulan más del $90 \%$ de los casos, mientras que colon derecho es excepcional, lo que es inverso a lo reportado por la experiencia asiática ${ }^{12,14-16}$. Cabe destacar que en nuestro estudio la localización exclusiva de divertículos en colon derecho fue más frecuente en el grupo menor de 60 años, dando fuerza a la teoría de su origen probablemente congénito. Si se considera la suma de divertículos exclusivos de colon derecho, más los casos pancolónicos, obtenemos una prevalencia de $7,8 \%$, lo que es menor a lo reportado previamente en población caucásica ${ }^{28,35}$. Nuestros casos del lado derecho están dados mayormente por los casos pancolonicos, y estos también aumentan con la edad, lo que contribuye a la idea de un ori-
Tabla 9. Distribución de los pacientes con divertículos según edad y sexo

\begin{tabular}{|cccc|}
\hline & $\begin{array}{c}\text { Total } \\
(\mathbf{n = 3 3 4})\end{array}$ & $\begin{array}{c}\text { Masculino } \\
(\mathbf{n}=\mathbf{1 5 4})\end{array}$ & $\begin{array}{c}\text { Femenino } \\
(\mathbf{n}=\mathbf{1 8 0})\end{array}$ \\
\hline$<60$ años & 130 & $69(53 \%)$ & $61(46,9 \%)$ \\
$>60$ años & 204 & $85(41,7 \%)$ & $119(58,3 \%)$ \\
\hline
\end{tabular}

La edad se muestra con punto de corte en los 60 años.

gen similar a la diverticulosis izquierda, más que congénita.

Según nuestros resultados el sexo no sería un factor relevante para la presencia de divertículos de forma concordante a estudios previos que no muestran diferencias respecto al sexo, sin embargo, en un análisis bivariado encontramos que hay predominio femenino en los pacientes con divertículos sobre los 70 años de edad y predominio masculino en aquellos bajo los 40 años de edad. Este hallazgo también ha sido reportado en un estudio multicéntrico italiano ${ }^{36}$.

Como grupo nos llama la atención que los hallazgos locales se asemejan a lo reportado en estudios internacionales de hace 10 años o más y no se correlacionan con las cifras actuales. Esto podría estar dado por un envejecimiento poblacional con distintas tasas de crecimiento, siendo nuestra población más joven que la europea actual. Otra posible causa es que el método diagnóstico aun difiera, no por el TC en sí mismo, sino por las indicaciones para realizarlo y el grupo de pacientes estudiados.

Nuestro trabajo tiene algunas limitaciones, una de ellas es la inclusión de pacientes que se realizan un estudio por otra causa, por lo que no se pueden definir como sanos o asintomáticos. Además, esta cohorte es elegida en un centro hospitalario privado 
urbano, por lo que no es necesariamente representativa de la población nacional, con posibles sesgos de nivel socioeconómico y de localidad (rural vs urbano). A pesar de estas limitaciones relevantes, se considera que el estudio constituye un aporte en cuanto a ser la primera aproximación nacional del dato y que es un precedente para continuar y ampliar el estudio de forma multicéntrica o a nivel nacional.

\section{Conclusiones}

Según los resultados reportados, es factible definir que la prevalencia global de diverticulosis colónica asintomática en población nacional sería de aproximadamente $30 \%$, con un claro predominio de ubicación en colon izquierdo, especialmente, sigmoides con o sin afección del colon descenden- te. La prevalencia, características de localización y edad de presentación se asemejan a la literatura occidental de la década pasada más que a reportes internacionales recientes. Si bien el estudio cuenta con limitaciones, se considera un aporte por su naturaleza original, que podría conducir a futuras investigaciones de prevalencia y tamizaje.

\section{Responsabilidades éticas}

Protección de personas y animales. Los autores declaran que para esta investigación no se han realizado experimentos en seres humanos ni en animales.

Confidencialidad de los datos. Los autores declaran que en este artículo no aparecen datos de pacientes.

Conflictos de interés: no hay.

\section{Referencias}

1. Parks T. Natural history of diverticular disease of the colon. A review of 521 cases. Br Med J. 1969;4:639-42.

2. Rafferty J, Shellito P, Hyman N, Buie W. Standards Committee of the American Society of Colon and Rectal Surgeons. Practice parameters for sigmoid diverticulitis. Dis Colon Rectum. 2006;49:939-44.

3. Peery AF, Barrett PR, Park D, Rogers A, Galanko J, Martin C. A high-fiber risk diet does not protect against asymptomatic diverticulosis. Gastroenterology. 2012;144:266-72.

4. Moreira V, López A. Enfermedad diverticular del colon. Rev esp enferm dig. 2005;97:458.

5. Slack W. La anatomía, la patología y algunas características clínicas de la divericulitis del colon. Br J Surg. 1962;50:185-90.

6. De Cecco CN, Ciolina M, Annibale B, Rengo M, Bellini D, Muscogiuri G. Prevalence and distribution of colonic diverticula assessed with CT colonography (CTC). Eur Radiol. 2016;26:639-45.

7. Gear J, Ware A, Fursdon P, Mann J, Nolan D, Brodribb A. Symptomless diverticular disease and intake of dietary fibre. Lancet. 1979;1:511-4

8. Lin $\mathrm{O}$, Soon M, Wu S, Chen Y, Hwang
K, Triadafilopoulos G. Dietary habits and right sided colonic diverticulosis. Dis Colon Rectum. 2000;43:1412-8.

9. Welch C, Allen A, Donaldson G. Una evaluación de la resección del colon para la diverticulitis del sigmoide. Ann Surg. 1953;138:332-43.

10. Manousos O, Truelove S, Lumsden K. Prevalence of colonic diverticulosis in general population of Oxford area. BMJ. 1976;3:762-3.

11. Hughes L. Postmortem survey of diverticular disease of the colon. Part I. Diverticulosis and diverticulitis. Gut. 1969;10:336-44.

12. Everhart J, Ruhl C. Burden of digestive diseases in the United States part II: lower gastrointestinal diseases. Gastroenterology. 2009;136:741-54.

13. Lohsiriwat V, Suthikeeree W. Pattern and distribution of colonic diverticulosis: analysis of 2877 barium enemas in Thailand. Gastroenterol. 2013;19:8709-13.

14. Hughes L. Postmortem survey of diverticular disease of the colon. Part II. The muscular abnormality of the sigmoid colon. Gut. 1969;10:344-51.

15. Stumf M, Cao W, Klinge U, Klosterhalfen B, Kasperk R, Schumpelick V. Increased distribution of collagen type III and reduced expression of matrix metalloproteinase 1 in patients with diverticular disease. Colorectal Dis. 2001;16:271-5.
16. Wess L, Eastwood M, Wess T, Busuttik A, Miller A. Cross linking of collagen is increased in colonic diverticulosis. Gut. 1995;37:91-4.

17. Strate L, Erichsen R, Baron J, Mortensen J, Pedersen J, Riis A, et al. Heritability and familial aggregation of diverticular disease: a population-based study of twins and siblings. Gastroenterology. 2013;144:736-42.

18. Yamada E, Inamori $M$, Uchida $E$, Tanida E, Izumi M, Takeshita, et al. Association between the localization of diverticular disease and the irritable bowel syndrome: a multicenter study in Japan. AM J Gastroenterol. 2014;109:1900-5.

19. Nuñez J, Albarracin J, Petricic B, Babieri $\mathrm{M}$, Soffia P. Colonoscopia virtual: realidad en un hospital público. Rev chil radiol. 2007;13:5-8.

20. Köhler L, Sauerland S, Neugebauer E. Diagnosis and treatment of diverticular disease: results of a consensus development conference. The Scientific Committee of the European Association for Endoscopic Surgery. Surg Endosc. 1999;13:430-6.

21. Derbyshire E, Hungin P, Nickerson C, Rutter M. Colonoscopic perforations in the English National Health Service Bowel Cancer Screening Programme. Endoscopy. 2018, DOI: 10.1055/a-05847138.

22. Ambrosetti P, Grossholz M, Becker C, 
Terrier F, Morel P. Computed tomography in acute left colonic diverticulitis. Br J Surg. 1997;84:532-4.

23. Flor N, Maconi G, Cornalba G, Pickhardt P. The Current Role of Radiologic and Endoscopic Imaging in the Diagnosis and Follow-Up of Colonic Diverticular Disease. AJR Am J Roentgenol. 2016;207:15-24.

24. Bannura G, Contreras J, Melo C, Barrera A, Soto D, Mansilla J. Indicaciones resultados alejados del tratamiento quirúrgico electivo de la enfermedad diverticular del colon sigmoides. Rev Med Chile. 2005;133:1037-42.

25. López-Köstner F, Zárate A, Pinedo G, Molina M, Kronberg U, Pardo J. Resultados de la cirugía laparoscópica en el tratamiento electivo de la enfermedad diverticular de colon. Rev Med Chile. 2008;136:594-9.

26. Loffeld RJ, Van der Putten AB. Diverticular disease of the colon and concomitant abnormalities in patients undergoing endoscopic evaluation of the large bowel. Colorectal Dis. 2002;4:18992.

27. Blachut K, Paradowski L, Garcarek J. Prevalence and distribution of the colonic diverticulosis. Review of 417 cases from Lower Silesia in Poland. Rom J Gastroenterol. 2004;13:281-5.

28. Manousos $\mathrm{O}$, Truelove $\mathrm{S}$, Lumsden $\mathrm{K}$. Transit times of food in patients with diverticulosis or irritable colon syndrome and normal subjects. Br Med J. 1967;3:760-2.

29. Azzam N, Aljebreen A, Alharbi O, Almadi A. Prevalence and clinical features of colonic diverticulosis in a Middle Eastern population. World J Gastrointest Endosc. 2013;5:391-7.

30. Golder M, Chis I, Babu P, Sharma A, Bayat M, Farah A. Demographic determinants of risk, colon distribution and density scores of diverticular disease. World J Gastroenterol. 2011;17(8):100917.

31. Pemberton J. Colonic diverticulosis and diverticular disease: Epidemiology, risk factors, and pathogenesis. UpToDate 2017.

32. Ouriel K, Schwartz S. Diverticular disease in the young patient. Surg Gynecol Obstet. 1983;156:1-5.

33. Biondo S, Parés D, Martí J, Kreisler E, Fraccalvieri D, Jaurrieta E. Acute colonic diverticulitis in patients under 50 years of age. BJS. 89:1137-41.

34. Afzal N, Thomson M. Diverticular disease in adolescence. Best Practice \& Research Clinical Gastroenterology. 2002;16:62134.

35. Roberts P, Abel M, Rosen L, Cirocco W, Fleshman J, Leff E, et al. Practice parameters for sigmoid diverticulitis. The Standards Task Force American Society of Colon and Rectal Surgeons. Dis Colon Rectum. 1995;38:125-32.

36. Annibale B, Lahner E, Maconi G, Usai P, Marchi S, Bossotti G, et al. Clinical features of symptomatic uncomplicated diverticular disease: a multicenter Italian survey. Int J Colorectal Dis. 2012;27:1151-9. 